\title{
An Index to Deoxyribonucleic Acid Base Compositions of Bacterial Species
}

\author{
By L. R. HILL \\ National Collection of Type Cultures, Central Public Health Laboratory, \\ Colindale Avenue, London
}

(Received 3 March 1966)

\begin{abstract}
SUMMARY
This paper consists of a reference list of bacterial species for which deoxyribonucleic acid base compositions are known. Culture-collection strain numbers have been included wherever possible. The compilation may also provide a basis for the inclusion of these data into species descriptions.
\end{abstract}

\section{INTRODUCTION}

Deoxyribonucleic acid (DNA) base compositions are becoming widely used in taxonomy and it is already evident that there is a need to include such data into future species descriptions. The more recent reviews (e.g. Sueoka, 1961; Marmur, Falkow \& Mandel, 1963; De Ley, 1964) omitted references to specific strains, whilst many reports of bacterial DNA base compositions have appeared since the last detailed review (Belozersky \& Spirin, 1960). A survey of the literature to the end of 1965 shows that DNA base compositions have already been reported for about 400 differently named species. Moreover, about half the entries in the present compilation of data from the literature refer to strains deposited in various culture collections.

The index is arranged as follows. In the first column the genera are arranged alphabetically and the species alphabetically within their genera. Generic and specific epithets used in the original papers are retained and synonyms quoted by the authors are given in parenthesis. The only exceptions are: 'Diplococcus' is included in Streptococcus; 'Coxiella' in Rickettsia; and 'Staphylococcus roseus' and 'Staphylococcus afermentans' are transferred to Micrococcus. In the second column Culture Collection numbers or other strain identification symbols are given wherever possible. Reference to the technique used for the determination of the base composition is given in the third column. The base compositions, expressed as moles $\%$ of guanine + cytosine ( $\%$ GC, hereafter) of the moles of total bases, are listed in column 4. Finally, the fifth column contains numerical references to the original papers. The references are arranged alphabetically and are numbered, these numbers being used in the index.

A graphical representation of the data is given in Figs. 1 and 2 which include only those genera for which at least two species or strains have been investigated for DNA base compositions. In the figures, the genera are arranged following the classification in Bergey's Manual (1957). Those genera which are not listed in the Manual are placed after the genus they most closely resemble. 
The author would greatly appreciate receiving notices of any errors or omissions that may have escaped him.

\section{Column 2. Strain number}

\section{Abbreviations used in the index}

A Amc American Type Culture Collection, Washington.

CCEB Culture Collection of Entomogenous Bacteria, Prague.

CCM Czechoslovak Collection of Micro-organisms, Brno.

IP Institut Pasteur, Paris.

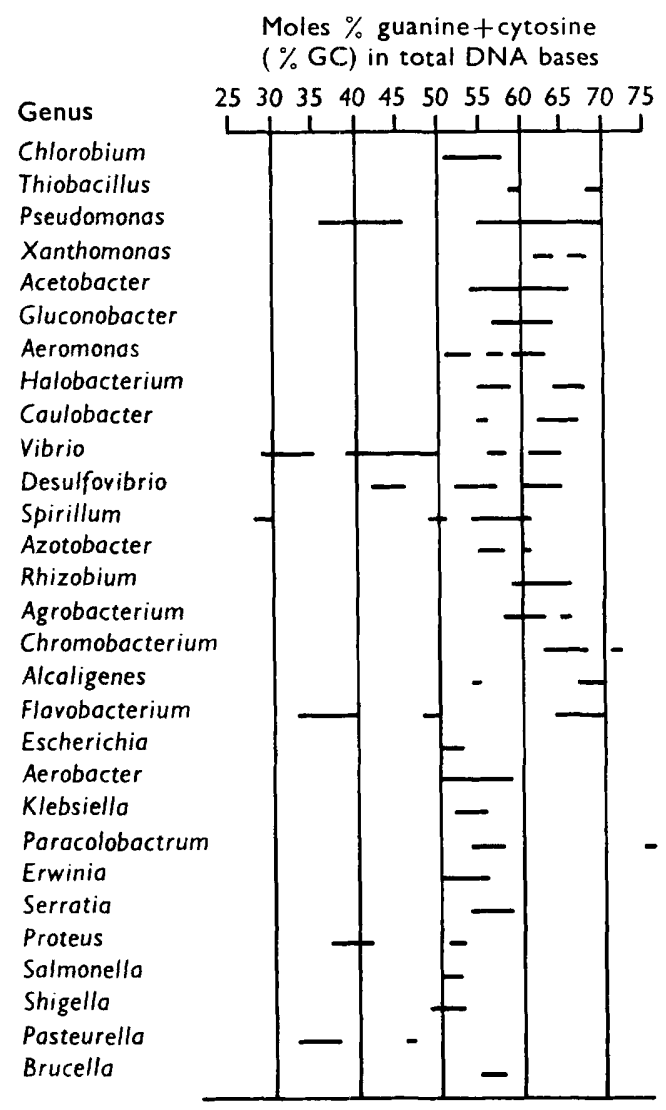

Fig. 1

\section{Genus}

Haemophilus

Moraxella

Bocteroides

Fusobacterium

Sphaerophorus

Micrococcus

Staphylococcus

Sarcina

Neisseria

Streptococcus

Lactobacillus

Bifidobacterium

Cotenabacterium

Propionibacterium

Corynebacterium

Arthrobacter

Bacillus

Clostridium

Mycobacterium

Nocardia

- Streptomyces

Thermoactinomyces

Streptosporangium

Cytophaga

Myxococcus

Leptospira

Mycoplasma

Rickettsia
Moles $\%$ guanine + cytosine

$(\% \mathrm{GC})$ in total DNA bases

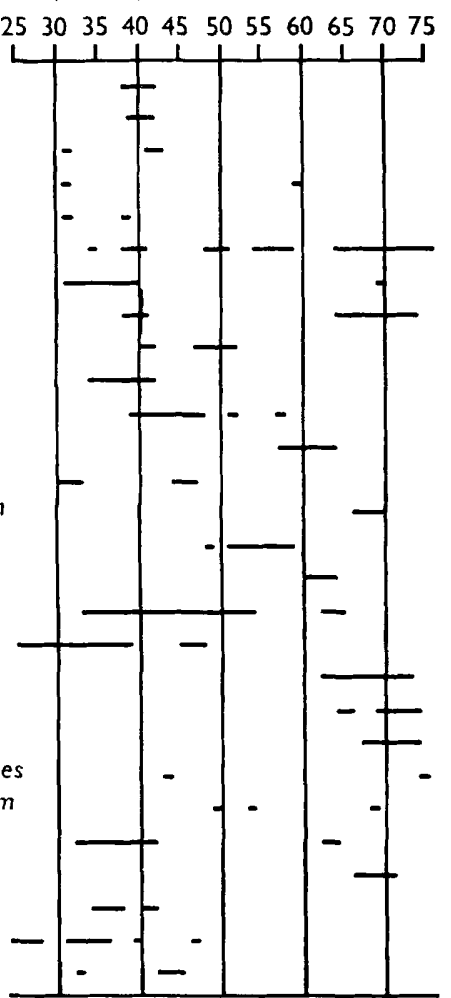

Fig. 2

Figs. 1 and 2. Graphical representation of the data given in the table. Only genera for which at least two species or strains have been investigated for DNA base composition are included. The horizontal lines represent the range of \% GC values reported for each genus.

IMRU Institute of Microbiology, Rutgers University, New Brunswick.

NCDo National Collection of Dairy Organisms, Shinfield, Reading, Berkshire.

NCIB National Collection of Industrial Bacteria, Aberdeen.

NCMB National Collection of Marine Bacteria, Aberdeen.

NCTC National Collection of Type Cultures, London.

NRRL Northern Utilization Research and Development Division, U.S. Department of Agriculture, Peoria. 


\section{Column 3. Method}

The various techniques used to determine DNA base composition have been categorized as follows:

a Methods of hydrolysis and chromatography, reviewed by Bendich (1957).

b 'Melting temperature' $(\mathrm{Tm})$ determinations from the sharp increase in absorbance at $250 \mathrm{~m} \mu$ upon heating dilute aqueous solutions of the DNA (Marmur \& Doty, 1962).

c Determinations of the buoyant density of DNA in caesium chloride equilibrium ultra-centrifugation (Schildkraut, Marmur \& Doty, 1962).

d Determinations of the ratio of absorbancies of DNA at 260 and $280 \mathrm{~m} \mu$ at $\mathrm{pH} 3$ (Fredericq, Oth \& Fontaine, 1961).

e Spectroscopic determinations of the relative percentage of adenine by its reactivity towards bromine reagents (Wang \& Hashagen, 1964).

Column 4. \% GC

To the nearest $0.5 \%$, the moles $\%$ guanine + cytosine $/$ moles total bases.

Table 1. Index to DNA base compositions of bacterial species: moles $\%$ guanine + cytosine $(\% G C)$ of total bases

\begin{tabular}{|c|c|c|c|c|}
\hline Species & Strain & Method & $\%$ GC & Ref. \\
\hline \multicolumn{5}{|l|}{ Acetobacter } \\
\hline aceti & Ch. 31 & $\mathrm{ab}$ & $59 \cdot 5$ & 18 \\
\hline aceti (liquefaciens) & NCIB 9505 & $\mathbf{a b}$ & $64-65 \cdot 5$ & 14,18 \\
\hline aceti (liquefaciens) & p $12 \alpha$ & b & $64 \cdot 5$ & 14 \\
\hline aceti var. muciparus & 5 & b & $59 \cdot 5$ & 18 \\
\hline ascendens & ATCC 9323 & b & 55 & 37 \\
\hline cerinus var. rosiensis & 22 & $\mathbf{a b}$ & $55 \cdot 5-56 \cdot 5$ & 18 \\
\hline estunensis & $\mathbf{E}$ & b & 62 & 18 \\
\hline gluconicum & $2 \mathrm{G}$ & be & $\mathbf{5 4 - 5 5}$ & 37,48 \\
\hline mesoxydans var. saccharovorans & - & $\mathbf{a b}$ & $60 \cdot 5-61$ & 18 \\
\hline mobilis & NCIB 6428 & $\mathbf{a b}$ & 59 & 18 \\
\hline paradoxus & P 1 & $\mathbf{b}$ & $\mathbf{5 5} \cdot 5$ & 18 \\
\hline paradoxus & $\mathbf{P} 2$ & $\mathbf{b}$ & $55 \cdot 5$ & 18 \\
\hline pasteurianum & 11 & b & 60 & 18 \\
\hline peroxydans & NCIB 8618 & $a b$ & $61-62 \cdot 5$ & 18 \\
\hline peroxydans & $\mathbf{3}$ & b & $63 \cdot 5$ & 18 \\
\hline peroxydans & 4 & b & $63 \cdot 5$ & 18 \\
\hline rancens & $23 \mathbf{k l}$ & $\mathbf{b}$ & $55 \cdot 5$ & 18 \\
\hline vini acetati & NCIB 4939 & b & $57 \cdot 5$ & 18 \\
\hline xylinoides & NCIB 4940 & b & $62 \cdot 5$ & 18 \\
\hline xylinum & NCIB 8747 & $\mathbf{a b}$ & 61 & 18 \\
\hline xylinum. & 25 & $\mathbf{b}$ & 63 & 18 \\
\hline Achromobacter fischeri & - & - & $40-42$ & 38 \\
\hline Actinobacterium abscessum & 1852 B & $\mathbf{a}$ & $61 \cdot 5$ & 49 \\
\hline Actinomyces propionici & - & a & $66 \cdot 5$ & 49 \\
\hline Actinoplanes philippinensis & - & $\mathbf{b}$ & 73 & 28 \\
\hline \multicolumn{5}{|l|}{ Aerobacter } \\
\hline aerogenes & (4 strains) & $\mathbf{a}$ & $57-59$ & $\mathbf{1}$ \\
\hline aerogenes & ATCC 14308 & be & 54 & 9 \\
\hline aerogenes & ATCC 13048 & be & $54 \cdot 5$ & $9, \mathbf{3 5}$ \\
\hline aerogenes & MEG & be & $\mathbf{5 4} \cdot 5$ & 9 \\
\hline aerogenes & ATCC 211 & be & $\mathbf{5 5} \cdot \mathbf{5}$ & 9 \\
\hline aerogenes & Indiana & be & $\mathbf{5 5 \cdot 5}$ & 9 \\
\hline aerogenes & Balamuth & bc & $56 \cdot 5$ & 9 \\
\hline
\end{tabular}




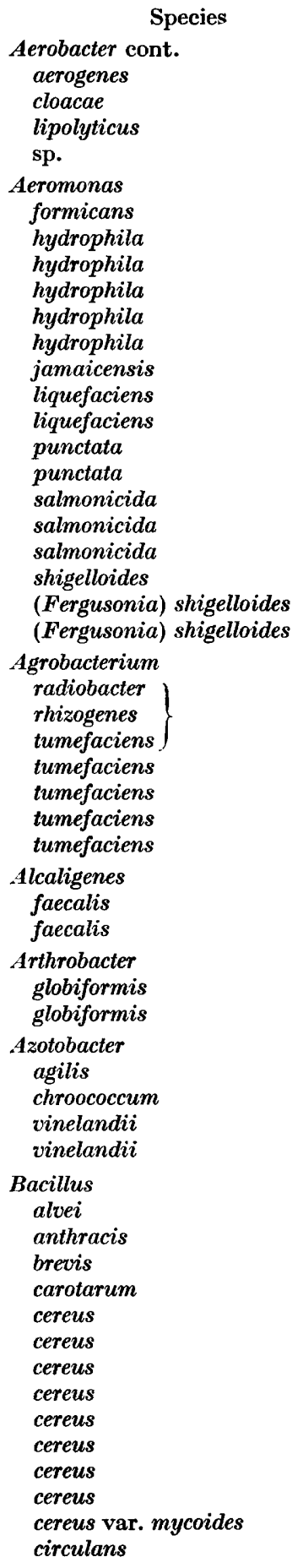

Table 1 (cont.)

\begin{tabular}{llll}
\multicolumn{1}{c}{ Strain } & Method & \multicolumn{1}{c}{$\%$ GC } & Ref. \\
& & & \\
1088 (Harvard) & bc & $57-57 \cdot 5$ & 37,48 \\
- & - & $52-54$ & 38 \\
$\overline{\text { 1041 }}$ & - & $50-52$ & 37 \\
& b & $51 \cdot 5$ & 37
\end{tabular}

$\begin{array}{llll}\text { ATCC 13137 } & \text { b } & 59 & 9 \\ \text { ATCC 9071 } & \text { b } & 55 \cdot 5 & 35 \\ \text { IP R 126 } & \text { a } & 59 & 50 \\ \text { IP R 307 } & \text { a } & 59 \cdot 5 & 50 \\ \text { NCTC 7810 } & \text { a } & 61 \cdot 5 & 50 \\ \text { NCTC 7812 } & \text { a } & 62 \cdot 5 & 50 \\ \text { Caselitz X3 } & \text { a } & 62 \cdot 5 & 50 \\ \text { NRRL B-966 } & \text { b } & 59 & 9 \\ \text { ATCC 14715 } & \text { b } & 59 & 9 \\ \text { ATCC 11163 } & \text { bc } & 56 \cdot 5 & 9 \\ \text { CCEB 386 } & \text { a } & 61 & 50 \\ \text { ATCC 14174 } & \text { b } & 56 \cdot 5 & 9 \\ \text { CCEB 382 } & \text { a } & 57 & 50 \\ \text { NCMB 833 } & \text { a } & 58 \cdot 5 & 50 \\ \text { ATCC 14029 } & \text { b } & 52 & 35 \\ \text { IP 633 } & \text { a } & 51 & 50 \\ \text { NCIB 9242 } & \text { a } & 52 \cdot 5 & 50\end{array}$

$\begin{array}{lll}\text { (c. } 30 \text { strains) } \quad-\quad 59-60 & 15\end{array}$

$\begin{array}{llll}\text { B } 6 & \text { b } & 61.5 & 14\end{array}$

$\begin{array}{llll}\text { s } 1 & \text { b } & 62 & 14\end{array}$

$\begin{array}{llll}\text { M } 39 & \text { b } & 65.5 & 14\end{array}$

$\begin{array}{llll}- & \text { a } & 58-59 & 1\end{array}$

$\begin{array}{llll}\text { ATCC } 8750 & \text { bc } & 55\end{array}$

$\begin{array}{llll}- & \mathrm{a} & 67-70 & 1\end{array}$

$\begin{array}{llll}- & -62-64 & 38\end{array}$

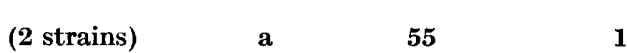

$\begin{array}{llll}\text { (4 strains) } & \text { a } & 57 \cdot 5 & 1\end{array}$

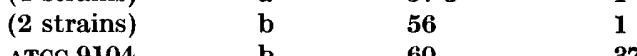

$\begin{array}{llll}\text { ATCC 9104 } & \text { b } & 60 & 37\end{array}$

$\begin{array}{llll}\text { ATCC 6344 } & \text { b } & 33 & 37\end{array}$

(2 strains) b $\quad 32-34 \quad 40$

$\begin{array}{llll}\text { ATCC } 9999 & \text { be } & 43-45 & 37,48\end{array}$

$\begin{array}{llll}- & \text { c } & 42 & 48\end{array}$

$\begin{array}{llll}\text { ATCC } 12137 & \text { a } & 36 & 1\end{array}$

(9 strains) $\quad$ a $\quad 33-37 \quad 1$

$\begin{array}{llll}\text { MB-19 be } & \mathbf{3 3 - 3 7} & \mathbf{3 7}, \mathbf{4 8}\end{array}$

$\begin{array}{llll}\text { ATCC 7064 } & \text { b } & 32 & 40\end{array}$

$\begin{array}{llll}\text { ATCC } 11950 & \text { b } & 32 & 40\end{array}$

$\begin{array}{llll}\text { ATCC } 9139 & \text { b } & 32.5 & 40\end{array}$

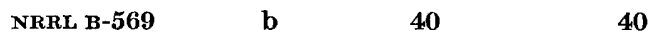

(2 strains) $\quad$ b $\quad 31 \cdot 5-33 \quad 40$

$\begin{array}{llll}75 \mathrm{a} & \text { c } & 39 & 48\end{array}$

$\begin{array}{llll}\text { ATCC } 4513 & \text { b } & 35 & 37\end{array}$ 
Table 1 (cont.)

Species

Strain

Method

$\% \mathbf{G C}$

Ref.

Bacillus cont.

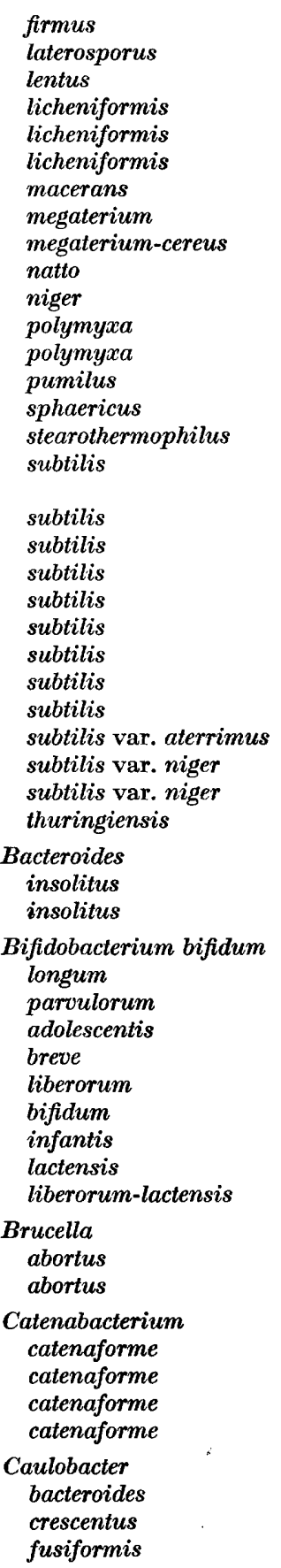

-
ATCC 64
ATCC 10840
ATCC 9789
ATCC $9945 a$
(2 strains)
ATCC 7069
-
-
MB 275
ATCC 6454
ATCC 842
NRRL B-367
ATCC 6631
ATCC 4525
194
168

b

$\begin{array}{lll}\text { b } & 41 & 37\end{array}$

$\begin{array}{lll}\text { b } & 37 & 37\end{array}$

abc $\quad 46-51 \quad 21,37,48$

b $\quad 44$

b 43

be $\quad 50-54$

abe $\quad 37-38$

b 34

be $\quad 43-44$

be $\quad 43$

b 44

c $\quad 47-48$

be $\quad 39-41$

b 37

bc 44-46

abcde 39-45

168 Mut мк9

168 Mut MK 12

168 Mut sc-22

-

ATCC 6633

ATCC 6051

(6 strains)

ATCC 6460

ATcc 6455

(Spores)

ATCC 10792

\section{abd}

63-64

abd 62-63

abd 64-65

a

43.5

$42 \cdot 5$

a

c

b

$\mathbf{b}$

b

abc

43

43

$45 \cdot 5-46 \cdot 5$

43

43

$42 \cdot 5$

34-36

40

40

37,48

$1,37,48$

37

37,48

37,48

37

20

20,37

37

37,48

$25,28,37$

$48,56,57$

25

25

25, 57

31

1

20

39

40

37

37

34

$1,37,48$

Is 9

NL 12

a

43

41

1

(6 strains)

(2 strains)

(5 strains)

(2 strains)

(2 strains)

(3 strains)

(1 strain)

(5 strains)

(3 strains)

a

$\begin{array}{ll}57-61 \cdot 5 & 49 \\ 58-59 \cdot 5 & 49 \\ 58 \cdot 5-62 & 49 \\ 58 \cdot 5-62 \cdot 5 & 49 \\ 59 \cdot 5-60 \cdot 5 & 49 \\ 61 \cdot 5-62 & 49 \\ 61 & 49 \\ 58 \cdot 5-64 & 49 \\ 59 \cdot 5-63 \cdot 5 & 49\end{array}$

$19 \mathrm{M}$

a

a

a

a

a

a

a

CB 6

KA 3

CB 37

$\mathbf{a}$

47

49

c $\quad 66$

66
62

45

$\begin{array}{ll}\text { c } & 62 \\ \text { c } & 67\end{array}$

45

45 
Table 1 (cont.)

Species

Strain

Method

$\%$ GC

Ref.

Caulobacter cont.

henrici
henrici
intermedius
leidyi (Asticcacaulis excentricus)
subvibrioides
vibrioides limonus

CB 4

KA 1

CB-G

KA 4

CB 79

CB 21

Cellulomonas biazotea

ATCC 486

$\begin{array}{lll}\text { c } & 62 & 45 \\ \text { c } & 65 & 45 \\ \text { c } & 65 & 45 \\ \text { c } & 55 & 45 \\ \text { c } & 67 & 45 \\ \text { c } & 64 & 45 \\ \text { b } & 75 & 28\end{array}$

Chlorobium

thiosulfatophilum

spp.

spp.

spp.

Chondromyces apiculatus

Chromatium sp.

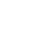

(6 strains)

bc

56-58

(3 strains)

(8 strains)

$\mathrm{K}$

be

51-52.5

53.5-55

be $\quad 56 \cdot 5-58$

c 70

64-66

Chromobacterium

lividum

lividum

lividum

lividum

lividum

violaceum

violaceum

violaceum

violaceum

violaceum

violaceum

Clostridium

acidiurici

bifermentans

butylicum

butylicum

chauvoei

cylindrosporum

kluyveri

madisoni

nigrificans

nigrificans

nigrificans

pasteurianum

perfringens

perfringens

saprogenes

tetani

tetanomorphum

valerianicum

NCTC 9796

NCTC 7150

NCIB 9230

GA

(7 strains)

NCTC 9373

NCTC 9371

NCTC 8684

NCTC 8685

NCTC 9757

NCrC 9694

$\begin{array}{lll}\text { b } & 65 \cdot 5 & 16 \\ \text { b } & 65 \cdot 5 & 16 \\ \text { b } & 65 \cdot 6-66 & 14 \\ \text { b } & 71 \cdot 5 & 16 \\ \text { b } & 66 & 14 \\ \text { b } & 63 \cdot 5 & 16 \\ \text { b } & 66 & 16 \\ \text { b } & 67 & 16 \\ \text { b } & 67 & 16 \\ \text { b } & 67 & 16 \\ \text { b } & 67 \cdot 5 & 16\end{array}$

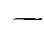

ATCC 862

$\begin{array}{lll}\text { abce } & 25-32 & 55 \\ \text { a } & 32 \cdot 5 & 1\end{array}$

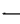

$-$

-

16

NCIB 8395

(2 strains)

(2 strains)

$\overline{87 \mathrm{~b}}$

(2 strains)

$\overline{1}$

Corynebacterium

acnes

anaerobium

anaerobium

annamensis

avidum

bovis

cutis commune

diphtheriae

diphtheroides

-

(2 strains)

38

1

$\begin{array}{lll}\text { c } & 38 & 48\end{array}$

ab $\quad 31 \cdot 5-37 \cdot 5 \quad 55$

be $\quad 26 \cdot 5-32 \quad 37,48$

abe $\quad 32-32.5 \quad 55$

$\begin{array}{lll}\text { c } & 35 & 48\end{array}$

bc $\quad 26 \cdot 5-34 \quad 37,48$

$\begin{array}{lll}\text { c } & 48 & 48\end{array}$

c 44.547

$\begin{array}{lll}\text { c } & 45.5 & 47\end{array}$

abce $\quad 30 \cdot 5-31.5 \quad 55$

bc $\quad 26 \cdot 5-32 \quad 37,48$

a $27-31 \quad 1$

$\begin{array}{lll}\text { a } & 30 \cdot 5 & 1\end{array}$

be $\quad 26 \cdot 5-34 \quad 37,48$

$\begin{array}{lll}\text { a } & 39 \cdot 5 & 55\end{array}$

$\begin{array}{lll}\text { a } & 32 & 1\end{array}$

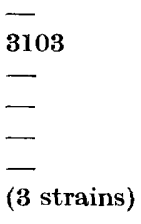

$\begin{array}{ll}48 & \mathbf{1}, 5 \\ \mathbf{5 1} \cdot 5 & \mathbf{5} \\ \mathbf{5 8} & \mathbf{4 9} \\ \mathbf{5 8} & \mathbf{5} \\ \mathbf{5 2} & \mathbf{5} \\ \mathbf{5 8} & 5 \\ \mathbf{5 4} & \mathbf{5} \\ \mathbf{5 2 - 5 4} \cdot 5 & \mathbf{1 , 5} \\ \mathbf{5 3} & \mathbf{5}\end{array}$


Table 1 (cont.)

Species

Corynebacterium cont.

enzymicum
equi
fascians
granulomatis
granulosum
hepatodystrophicans
hoagii
hofmannii
humiferum
kutscheri
liquefasciens
lymphophilum
michiganense
minutissimum
parvum
parvum
phocae
pseudotuberculosis
pyogenes
rathayi
renale
renale cuniculi
vadosum
xerosis
xerosis
sp.
sp.
sp.

sp.

Cytophaga aurantiaca fermentans hutchinsonii johnsonii johnsonii

sp.

sp.

sp.

sp.

sp.

Desulfovibrio aestuarii desulfuricans desulfuricans desulfuricans desulfuricans desulfuricans desulfuricans desulfuricans desulfuricans desulfuricans desulfuricans desulfuricans desulfuricans

desulfuricans desulfuricans desulfuricans

Strain Method

$\% \mathrm{GC}$

Ref.

-
-
-
-
-
-
-
-
-
-
-
936 B
-
-
-
-
-
ATCC 9016
с 85 2
CT 2
-

$51 \cdot 5$

$58 \cdot 55$

54.55

$51 \cdot 5 \quad 5$

525

$52 \cdot 5$

51.55

575

54.5 5

58.55

$51 \cdot 5$

545

$53.5 \quad 5$

$54 \cdot 5$

5819

$54.5 \quad 49$

525

52.55

$48 \cdot 5$

545

$53 \quad 5$

$58 \quad 5$

57.51

55 5

$57 \cdot 5-59 \quad 37,48$

535

$53 \quad 5$

$55.5 \quad 5$

ATCC 12208

ATCC 12470

MYX 1.1.1

Cook 405

NCMB 11

NCMB 292

NCMB 275

NCMB 244

NCMB 259

NCIB 8380

NCIB 8303

NCIB 8319

NCIB 9442

NCIB 8311

NCIB 8386

NCIB 8305

NCIB 8302

NCIB 8446

NCIB 8387

NCIB 8401

NCIB 9335

'V. cholinicus' ATCC 13541

NCIB 8310

NCIB 8312

NCrB 8391 
Table 1 (cont.)

Species

Desulfovibrio cont. desulfuricans

desulfuricans

desulfuricans

desulfuricans

desulfuricans

desulfuricans

desulfuricans

desulfuricans

desulfuricans

desulfuricans

desulfuricans

desulfuricans

desulfuricans

orientis

orientis

sp.

sp.

Erwinia

ananas
aroideae
carotovora
carotovora
carotovora
carotovora
carotovora
carotovora
carotovora
chrysanthemi
cypripedi
maydis
milletiae
nigrificans
nimipressurabilis
rhapontica

Escherichia

aurescens

coli

(Citrobacter) freundii

Many strains ATcc 9723d

including $\left\{\begin{array}{l}\text { ATCC } 4157 \\ 5 \text { T }\end{array}\right.$

intermedium

Flavobacterium

acidificum

aquatile

arborescens

esteroaromaticum

flavescens

odoratum

suaveoleus

suaveoleus

vitarumens

vitarumens

sp.

sp.

sp.

sp.
Strain

NCIB 8363

NCIB 8388

NCIB 8393

ATCC 7757

NCIB 8380

NCIB 8387

NCIB 8307

NCIB 8322

NCIB 8398

NCIB 8403

NCIB 8365

NCIB 8364

NCIB 8308

NCIB 8382

NCIB 8445

Gigas

NCIB 8395

-
-17
64
51
58
$3 a$
ATCC 8061

一

二

-

二

-

5610-52

$$
-
$$

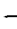

ATCC 11947
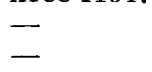

$-$

ATCC 958

ATCC 10234

NCMB 251

NCMB 289

NСMB 264

NCMB 249
Method

$\% \mathbf{G C}$

Ref.

$\begin{array}{lll}\text { c } & 55 \cdot 5 & 47 \\ \text { c } & 55 \cdot 5 & 47 \\ \text { ac } & \mathbf{5 5 \cdot 5}-57 \cdot 5 & 47,51 \\ \text { c } & 55 \cdot 5 & 47 \\ \text { abc } & 52 \cdot 5-56 & 47,51 \\ \text { c } & 54 \cdot 5 & 47 \\ \text { c } & 54 \cdot 5 & 47 \\ \text { c } & 54 \cdot 5 & 47 \\ \text { c } & 46 \cdot 5 & 47 \\ \text { bc } & 44-45 \cdot 5 & 47 \\ \text { c } & 45 \cdot 5 & 47 \\ \text { c } & 45 \cdot 5 & 47 \\ \text { c } & 45 \cdot 5 & 47 \\ \text { ac } & 41 \cdot 5-42 & 47,51 \\ \text { c } & 41 \cdot 5 & 47 \\ \text { a } & 64 \cdot 5 & 51 \\ \text { a } & 45 \cdot 5 & 51\end{array}$

$\begin{array}{lll}- & \mathbf{5 2 - 5 4} & \mathbf{3 8} \\ - & \mathbf{5 0 - 5 2} & \mathbf{3 8} \\ \mathbf{a} & \mathbf{5 5} & \mathbf{2 2} \\ \text { a } & \mathbf{5 3 \cdot 5} & \mathbf{2 2} \\ \text { a } & \mathbf{5 5} & \mathbf{2 2} \\ \text { a } & \mathbf{5 4} & \mathbf{2 2} \\ \text { a } & \mathbf{5 3 \cdot 5} & \mathbf{2 2} \\ \text { bc } & \mathbf{5 0 - 5 1 \cdot 5} & \mathbf{3 7}, \mathbf{4 8} \\ \text { a } & \mathbf{5 4} & \mathbf{1} \\ - & \mathbf{5 2 - 5 4} & \mathbf{3 8} \\ - & \mathbf{5 2 - 5 4} & \mathbf{3 8} \\ - & \mathbf{5 0 - 5 2} & \mathbf{3 8} \\ - & \mathbf{5 2 - 5 4} & \mathbf{3 8} \\ - & \mathbf{5 4 - 5 6} & \mathbf{3 8} \\ - & \mathbf{5 2 - 5 4} & \mathbf{3 8} \\ - & \mathbf{5 0 - 5 2} & \mathbf{3 8}\end{array}$

$\begin{array}{lll}- & 50-52 & 38 \\ - & 50-52 & 1,9,35,55 \\ \text { bc } & 50-53 & 37,48 \\ - & 50-52 & 38\end{array}$

-- $48-50 \quad 38$

$\begin{array}{lll}\text { bc } & \mathbf{3 2} \cdot 5 & 9\end{array}$

- $\quad 66-68 \quad 38$

$68-70 \quad 38$

66-68 38

34-38 38

66-68 38

$64.5 \quad 9$

64-66 $\quad 38$

$63.5 \quad 9$

$35 \cdot 5 \quad 16$

3616

$36 \cdot 5 \quad 16$

40.516 
Table 1 (cont.)

\section{Species}

Fusobacterium

fusiforme

polymorphum

Gluconobacter

capsulatus

gluconicum

melanogenus

melanogenus

melanogenus

suboxydans

suboxydans

suboxydans

viscosus

Haemophilus

aegypti

gallinarum

influenzae

influenzae

influenzae

parainfluenzae

suis

Halobacterium

cutirubrum

salinarum

salinarum

sp.

spp.

sp.

Klebsiella

aerogenes

atlantae

edroardsii

pneumoniae

pneumoniae

rhinoscleromatis

Lactobacillus

acidophilus

bifidus

brevis

buchneri

casei var. alactosus

case $i$ var. casei

casei var. rhamnosus

cellobiousus

fermenti

plantarum

Leptospira
biflexa
pomona

Leuconostoc mesenteroides

Listeria monocytogenes

Methanobacterium omelianski
Strain

Method

$\% \mathbf{G C}$

Ref. a

a

ab

b

ab

b

b

b

$\mathrm{ab}$

ab

ab

NCIB 8131

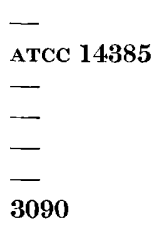

(2 bands)

(2 bands)

ССм 2090

IE 1

IV A 2, IV A3

II G2

bc
b
ad
e
bc
be
b

b

c

bd

c

c

c

-

8821/

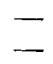

NCDO 473

NCDO 110

NCDO 680

NCDO 151

NCDO 243

NCDo 927

NCDO 215

NCDO 343

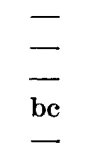

-

be

a

b

b

b

b

b

b

b

$\begin{array}{ll}- & - \\ - & - \\ \text { ATcC 12291 } & \text { bc } \\ - & \text { bc } \\ - & \text { a }\end{array}$
$31 \cdot 5$

60

1

1

$\begin{array}{ll}61 \cdot 5-62 & 18 \\ 61 \cdot 5 & 18 \\ 60 \cdot 5-61 & 18 \\ 60 \cdot 5 & 18 \\ 61 & 18 \\ 61 \cdot 5 & 18 \\ 57-58 & 18 \\ 61-62 & 18 \\ 62-63 \cdot 5 & 18\end{array}$

$\begin{array}{ll}39-40 & 37,48 \\ 42 & 46 \\ 38 & 1,23 \\ 41 & 56 \\ 39 & 37,48 \\ 39 & 37,48 \\ 39 & 37\end{array}$

58-59, $68 \quad 29$

$58,67 \quad 29$

654

$55 \quad 29$

$58 \quad 29$

$64 \quad 29$

52-54 38

52-54 38

$52-54 \quad 38$

55-56 37,48

$55 \quad 39$

$54-56 \quad 38$

$39-42 \quad 37,48$

$57 \cdot 5 \quad 1$

$45 \cdot 5 \quad 6$

446

$47 \cdot 5 \quad 6$

$46 \quad 6$

$47 \quad 6$

$45 \cdot 56$

51.56

436

$40-42 \quad 38$

34- $-38 \quad 38$

39-42 37,48

$38 \quad 37,48$

$44 \quad 55$ 
Table 1 (cont.)

\begin{tabular}{|c|c|c|c|c|}
\hline Species & Strain & Method & $\% \mathrm{GC}$ & Ref \\
\hline \multicolumn{5}{|l|}{ Micrococcus } \\
\hline (S.) afermentans & NCTC 7563 & $\mathbf{b}$ & 73 & 52 \\
\hline (S.) afermentans & NCTC 7503 & $a b$ & $72-74.5$ & 25 \\
\hline (S.) afermentans Mut. 44 & NeTC 7503 & $\mathrm{ab}$ & $72-73$ & 25 \\
\hline (S.) afermentans Mut. 22 & NCTC 7503 & abd & $68-70$ & 25 \\
\hline (S.) afermentans & NCTC 2665 & b & 73 & 52 \\
\hline aquivivus & CCM 316 & bd & $47 \cdot 5-51$ & 4 \\
\hline asaccharolyticus & - & - & 34 & 1 \\
\hline conglomeratus & $\operatorname{CCM} 740$ & bd & $53 \cdot 5-58 \cdot 5$ & 4 \\
\hline conglomeratus & CCM 2087 & bd & $64-68 \cdot 5$ & 4 \\
\hline conglomeratus & CCM 825 & $\mathbf{b}$ & $69 \cdot 5$ & 4 \\
\hline cryophilus & - & - & $38-40$ & 38 \\
\hline (Pediococcus) cryophilus & CCM 900 & bd & $41 \cdot 5-44 \cdot 5$ & 4 \\
\hline denitrificans & ССM 982 & bd & 64 & 4 \\
\hline denitrificans & CCM 1396 & bd & $66 \cdot 5-67$ & 4 \\
\hline eucinetus & CCM 2388 & bd & $47 \cdot 5-48$ & 4 \\
\hline eucinetus & CCM 2389 & bd & $49-50 \cdot 5$ & 4 \\
\hline halodenitrificans & $\operatorname{CCM} 286$ & bd & $65-66$ & 4 \\
\hline halodenitrificans & - & - & $64-66$ & 38 \\
\hline litoralis & CCM 2226 & bd & $63 \cdot 5-65$ & 4 \\
\hline luteus & CCM 132 & bd & $67-71$ & 4 \\
\hline luteus & CCM 840 & bd & $71-72$ & 4 \\
\hline luteus & CCM 1674 & bd & $71-71 \cdot 5$ & 4 \\
\hline luteus & CCM 337 & bd & $70-72 \cdot 5$ & 4 \\
\hline luteus & CCM 169 & b & $73 \cdot 5$ & 4 \\
\hline luteus & CCM 410 & bd & $70-73 \cdot 5$ & 4 \\
\hline luteus & CCM 149 & bd & $73-74 \cdot 5$ & 4 \\
\hline luteus & CCM 409 & bd & $72-75.5$ & 4 \\
\hline lysodeikticus & - & $\mathbf{a}$ & 72 & 1 \\
\hline lysodeikticus & - & $\mathrm{e}$ & 72 & 56 \\
\hline lysodeikticus & NRRL B287 & bc & 72 & 37,48 \\
\hline radiodurans & CCM 1700 & bd & $66-69$ & 4 \\
\hline radiodurans & CCM 1701 & bd & $65 \cdot 5$ & 4 \\
\hline radiodurans & - & $\mathbf{a}$ & 67 & 42 \\
\hline (S.) roseus & NCTC 7511 & b & 75 & 52 \\
\hline (S.) roseus & NCTC 7512 & $\mathbf{b}$ & 73 & 52 \\
\hline (S.) roseus & CCM 679 & bd & $68-69$ & 4 \\
\hline$(S)$. roseus & CCM 837 & bd & 70 & 4 \\
\hline (S.) roseus & CCM 385 & bd & $68-71$ & 4 \\
\hline (S.) roseus & CCM 560 & bd & $70-71$ & 4 \\
\hline (S.) roseus & CCM 633 & bd & $69-73$ & 4 \\
\hline sp. & $\operatorname{CCM} 168$ & bd & $45-49 \cdot 5$ & 4 \\
\hline sp. & CCM 1405 & bd & $49 \cdot 5-50 \cdot 5$ & 4 \\
\hline Microcyclus sp. & - & - & $64-66$ & 38 \\
\hline Micromonospora coerulea & - & $\mathbf{a}$ & 72 & I \\
\hline \multicolumn{5}{|l|}{ Moraxella } \\
\hline bovis & - & b & 41 & 37 \\
\hline liquefaciens & - & - & $40-42$ & 38 \\
\hline lwooffii & IP 55112 & $\mathbf{a}$ & $39 \cdot 5$ & 50 \\
\hline sp. (Vibrio sp. 01) & NCIB 8250 & a & 39 & 50 \\
\hline \multicolumn{5}{|l|}{ Mycobacterium } \\
\hline phlei & (2 strains) & $\mathbf{a}$ & $66 \cdot 5-68 \cdot 5$ & 1 \\
\hline phlei & - & be & $66-73$ & 37,48 \\
\hline smegmatis & - & - & 70 & 38 \\
\hline tuberculosis & (6 strains) & $\mathbf{a}$ & $62-70$ & 1 \\
\hline
\end{tabular}


Table 1 (cont.)

\begin{tabular}{|c|c|c|c|c|}
\hline Species & Strain & Method & $\%$ GC & Ref. \\
\hline \multicolumn{5}{|l|}{ Mycoplasma } \\
\hline gallinarum & ATCC 15319 & b & 28 & 46 \\
\hline gallisepticum & PPLO A5969 & bc & $34-35$ & 37,48 \\
\hline gallisepticum & PPLO A5969 & ac & $33-33 \cdot 5$ & 41 \\
\hline gallisepticum & ATCC 15302 & b & $32 \cdot 5$ & 46 \\
\hline hominis & 07 & a & $46 \cdot 5$ & 30 \\
\hline laidlaweii & Sewage $\mathbf{A}$ & be & $31-35 \cdot 5$ & 43 \\
\hline mycoides var. capri & PG 3 & a & $24-26$ & 26,27 \\
\hline pneumoniae & - & be & $39-41$ & 43 \\
\hline sp. & Calf & be & $23 \cdot 5-26 \cdot 5$ & 43 \\
\hline sp. & Goat-kid & be & 24-25.5 & 43 \\
\hline \multicolumn{5}{|l|}{ Myxососсиs } \\
\hline fulvus & (2 strains) & be & $69-71$ & 33 \\
\hline virescens & (4 strains) & bc & $68-71$ & 33 \\
\hline xanthus & (2 strains) & bc & $68-71$ & 33 \\
\hline xanthus & - & b & $68 \cdot 5$ & 28 \\
\hline spp. & (2 strains) & c & 69 & 33 \\
\hline \multicolumn{5}{|l|}{ Neisseria } \\
\hline catarrhalis & $\mathrm{Ne} 11$ & $\mathbf{a}$ & $40 \cdot 5$ & 7 \\
\hline catarrhalis & $\mathrm{Ne} 13$ & abc & $40-42$ & $7,37,48$ \\
\hline flava & $\mathrm{JJ} I \mathbf{I I}_{\mathbf{A}}$ & a & $49 \cdot 5$ & 7 \\
\hline flavescens & ATCC 13120 & abe & $47-49 \cdot 5$ & $7,37,48$ \\
\hline gonorrhoeae & - & a & $49 \cdot 5$ & 1 \\
\hline meningitidis & $\mathrm{Ne} 15$ & $a b c$ & $50-51 \cdot 5$ & $7,37,48$ \\
\hline meningitidis & - & a & 50.5 & 1 \\
\hline perflava & $\mathrm{Ne} 16$ & abc & $48-50 \cdot 5$ & $7,37,48$ \\
\hline perflava & $\mathrm{Ne} 20$ & a & 50 & 7 \\
\hline sicca & $\mathrm{Ne} 12$ & abe & $49-51 \cdot 5$ & $7,37,48$ \\
\hline subflava & ATCC 11076 & $\mathbf{a}$ & $50 \cdot 5$ & 7 \\
\hline Nitrosomonas sp. & - & 一 & $54-56$ & 38 \\
\hline \multicolumn{5}{|l|}{ Nocardia } \\
\hline asteroides & 92 & b & 66 & 28 \\
\hline asteroides & 96 & b & 64 & 28 \\
\hline asteroides & IMRU 727 & c & $69 \cdot 5$ & 24 \\
\hline brasiliensis & 301. & b & 65 & 28 \\
\hline citreus & - & $\mathbf{a}$ & 72 & 1 \\
\hline farcinica & 2089 & $\mathrm{~b}$ & $68 \cdot 5$ & 28 \\
\hline Orskov's motile nocardia & ATCC 12288 & b & 74 & 28 \\
\hline \multicolumn{5}{|l|}{ Paracolobactrum } \\
\hline aerogenoides & мек & be & $54-55$ & 37,48 \\
\hline (B) paracoli & - & $a b$ & 54-58 & $\mathbf{2 5}$ \\
\hline (B) paracoli & Mut. 52-1 & $a b$ & $75 \cdot 5$ & 25 \\
\hline (B) paracoli & Mut. cU-15 & b & $\mathbf{5 4} \cdot \mathbf{5}$ & 25 \\
\hline (B) paracoli & Mut. BU-12 & b & 54 & 25 \\
\hline \multicolumn{5}{|l|}{ Pasteurella } \\
\hline aviseptica & - & $\mathbf{a}$ & $36 \cdot 5$ & 1 \\
\hline boviseptica & - & a & $37 \cdot 5$ & 1 \\
\hline pestis & Ev 79 & c & 47 & 48 \\
\hline pestis & Avo2 & b & 46 & 37 \\
\hline pestis & Ev6 & b & 46 & \\
\hline tularensis & - & abc & $33-36$ & $1,37,48$ \\
\hline Pediococcus cerevisiae & CCM 833 & bd & $44 \cdot 5$ & 4 \\
\hline Polyangium cellulosum & - & - & $68-70$ & 38 \\
\hline
\end{tabular}


Species

Propionibacterium arabinosum freudenreichii pentosaceum shermanii technicum thoenii

zeae

\section{Proteus}

mirabilis

mirabilis

mirabilis $\mathbf{L}$ form

morganii

morganii

rettgeri

vulgaris

vulgaris

Providencia group

Pseudomonas

acidovorans

acidovorans

acidovorans

aeruginosa

aeruginosa

aeruginosa

aeruginosa

atlantica

chlororaphis

chlororaphis

cruciviae

cruciviae

denitrificans

desmolytica

diminuta

diminuta

fluorescens

fluorescens

fluorescens

fluorescens

fluorescens

fluorescens

fluorescens

fragi

geniculata

iodinum

iodinum

iodinum

indoloxidans

maltophilia

maltophilia

maltophilia

maltophilio

marginalis

oleovorans

ovalis

piscicida

piscicida

piscicida

Table 1 (cont.)

Strain

NCIB 5958

NCrB 5959

NCIB 8070

NCIB 8099

NCIB 5965

NCIB 8072

NCIB 8100

35

ATCC 14273

ATCC 14166

ATCC 8019

3478

(2 strains)

A TCC 9484

$$
\text { - }
$$

ATCC $\mathbf{1 5 6 6 6}$

ATCC 15667

ATCC 15668

NRRL B23

ATCC 8707

ATCC 8689

(4 strains)

NRRL B 560

NRRL B977

ATCC 13262

ATCC 12133

ATCC 15005

ATCC 11568

ATCC 949

ATCC 12121

ATCC 10796

ATCC 12633

ATCC 13034T

CCEB 448

B/s 1000

ATCC 4973

СCEB 338

ATCC 15729

ATCC 15728

ATCC 9897

ATCC 9355

ATCC 13843

ATCC 13636

ATCC 13637

ATCC 10858

ATcc 950

(1 strain)

(5 strains)

(7 strains)
Method

$\%$ GC

Ref.

$\mathbf{a}$
$\mathbf{a}$
$\mathbf{a}$
$\mathbf{a}$
$\mathbf{a}$

70.5

67

68

67

66.5

$67 \cdot 5$

$67 \cdot 5$

49

49

49

49

$4 \dot{9}$

49

49

$38-41$

$39 \cdot 5$

$39 \cdot 5$

53

51-51.5

$39 \cdot 5-42$

$36 \cdot 5-40 \cdot 5$

37-39

40-42

37,48

46

46

1

37,48

37,48

1

37, 48

be

38

bc

be

bc

bc

be

b

a

c

be

bc

be

be

be

-

c

be

b

b

bc

bc

b

a

be

b

bc

be

be

be

c

c

c

-

bc

bc

$60 \cdot 5-62$
$61 \cdot 5$
$64-64 \cdot 5$
$66-68$
64
$64 \cdot 5$
$64-67$
55

64

61

40-42

36-37.5

$56 \cdot 5-57 \cdot 5$

64.5-66

66-68

64.5

60-62

64

64.5

61

$63 \cdot 5-64 \cdot 5$

$62 \cdot 5$

63-64

57 5-62

$67 \cdot 5$

61-62.5

$61 \cdot 5-62 \cdot 5$

63.5

$64 \cdot 5-66 \cdot 5$

64.5

64.5

64.5

66-68

$57 \cdot 5-58 \cdot 5$

60-62

$64 \cdot 5$

$43 \cdot 5$

44

44.5
8

8

8

9

1

10

9

9

38

8

8

8

38

8

37,48

9

9

9

8

1

8

16

8

8

8

8

8

8

8

38

8

38

8

36

36

36 
Table 1 (cont.)

Species

Pseudomonas cont.

piscicida

piscicida

piscicida

putida

putida

putida

putrefaciens

reptilovora

saccharophila

stutzeri

syncyanea

labaci

testosteroni

sp.

Ramibacterium ramosum

Rhizobium

japonicum

spp. Peritrichous flagellation spp. Subpolar flagellation

Rhodospirillum rubrum

Rickettsia

burneti

burneti

prowazekii

Ristella (Bacteroides) clostridiformis

Salmonella

abony

arizona

ballerup

enteritidis

gallinarum

paratyphi $\mathbf{A}$

typhi

typhi

Saprospira grandis

Sarcina

aurantiaca

flava

flava

lutea

lutea

lutea

(Sporosarcina) ureae

(Sporosarcina) ureae

(Planosarcina) ureae

Serratia

indica

kiliensis

marinoruber

marcescens

marcescens

marcescens

marcescens

marcescens

\section{Strain}

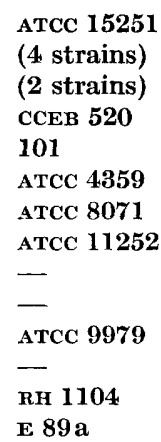

E9a

555

(20 strains)

(15 strains)

ATCC 11170

Paretsky
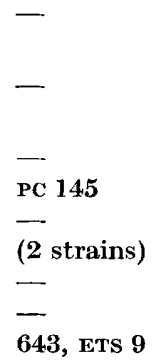

-

CCM 836

ATCC 540

26c

(2 strains)

C1

752

-

A'TCC 4003

ATCC 992

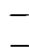

ATCC 4261

(3 strains)

ATCC 4013
Method

$$
\% \mathbf{G C}
$$

Ref.

$\begin{array}{ll}44 \cdot 5 & 36 \\ 45 & 36 \\ 45 \cdot 5 & 36 \\ 64 & 16 \\ 63 \cdot 5 & 16 \\ 58 \cdot 5-59 \cdot 5 & 8 \\ 43 \cdot 5-45 \cdot 5 & 8 \\ 61 \cdot 5-64 & 8 \\ 68-70 & 38 \\ 64 \cdot 5 & 8 \\ 61 \cdot 5-62 \cdot 5 & 8 \\ 67 \cdot 5 & 1 \\ 59 \cdot 5-60 \cdot 5 & 8 \\ 59 \cdot 5-61 & 8 \\ 30 & 1\end{array}$

bc

b

b

be

61-63

59-63

61-5-65.5

60-67

a

abc

44.5

42-45

$32 \cdot 5$

a

31

1

50-52

50-53

51.5

50

50

50

50-52

53

44-46

37, 48

17

17

37, 48

53

$37,48,58$

58

38

37,48

37

1

1

1

37,48

1

38

64-68.5 4

$70 \quad 31$

68.5 1

68-72 37,48

$72-74 \quad 1$

64: 1

$40-41 \cdot 5 \quad 31$

$40 \quad 31$

$\begin{array}{ll}38-40 & 38\end{array}$

$\begin{array}{ll}58 & 11 \\ 53 \cdot 5 & 11 \\ 56 \cdot 5 & 11 \\ 54 & 11 \\ 55 & 11 \\ 55 \cdot 5 & 11 \\ 56 \cdot 5 & 11 \\ 57 & 11\end{array}$


Table 1 (cont.)

Serratia cont.

\section{Species}

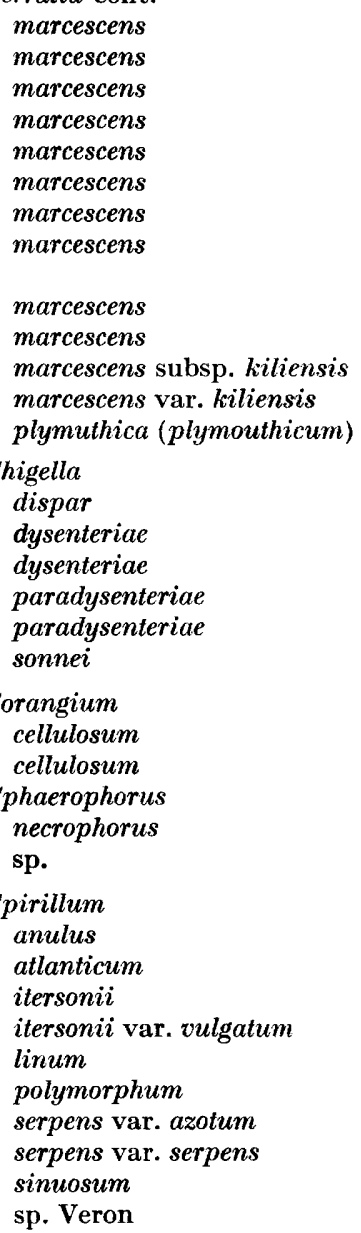

Sporocytophaga myxococcoides

Staphylococcus

aureus
aureus
aureus
aureus
aureus
aureus
aureus
aureus
aureus
aureus (coagulase -)
aureus (coagulase -)
aureus (coagulase -)
aureus (coagulase -)
lactis
lactis
lactis

Strain

(5 strains)
ATCC 13880
-
(6 strains)
ATCC 274
-
ATCC 990
ATCC 990
variant
(5 strains)
-
ATCC 8101
ATCC 11111
ATCC 183

15

15

Flexner y 6 R0

Flexner y 6 R 4

$$
-
$$

PY

$\mathbf{P X}$

$-$

NCMB 55

NCIB 9071

NCIB 9072

NCIB 9011

NCIB 8658

NCIB 9010

63 D

(2 strains)

ATCC 6538P

ATCC 14778

NCTC 8345

NCTC 4163

NCTC 8532

NCTC 6571

NCTC 4136

(5 strains)

NRRL $B 313$

CCM 313

CCM 641

CCM 127

CCM 529

NCTC 189

NCTC 7944

NCTC 7564
Method

$\%$ GC

Ref.

$\begin{array}{lll}\text { b } & 57 & 11 \\ \text { b } & 57 \cdot 5 & 11 \\ \text { bc } & 57 \cdot 5-59 & 37,48 \\ \text { b } & 58 & 11 \\ \text { b } & 58 & 11 \\ \text { a } & 58 & 1 \\ \text { b } & 58 \cdot 5 & 11 \\ \text { b } & 58 \cdot 5 & 11 \\ \text { b } & 58 \cdot 5 & 11 \\ \text { a } & 59 & 1 \\ \text { b } & 58 \cdot 5 & 11 \\ \text { b } & 59 & 11 \\ \text { b } & 57 \cdot 5 & 11 \\ & & \\ - & 50-52 & 38 \\ \text { a } & 53 \cdot 5 & 1 \\ \text { bc } & 50-51 & 37,48 \\ \text { a } & 49 \cdot 5 & 1 \\ \text { a } & 49 & 1 \\ \text { a } & 50-52 & 38\end{array}$

c

$69 \quad 33$

$69 \quad 33$

$31 \quad 19$

$38 \quad 19$

$54-56 \quad 38$

$50 \cdot 5 \quad 50$

54-56 38

$57 \quad 50$

28-30 $\quad 38$

$61.5 \quad 50$

$54 \quad 50$

$49 \quad 50$

61.550

6150

$36 \quad 33$

$\begin{array}{ll}37 \cdot 5 & 1 \\ 31 & 1 \\ 31 \cdot 5 & 1 \\ 31 & 52 \\ 31 & 52 \\ 32 & 52 \\ 33 & 52 \\ 30 \cdot 5-40 & 1 \\ 34 & 37,48 \\ 30-32 \cdot 5 & 4 \\ 33 \cdot 5-35 & 4 \\ 30-34 & 4 \\ 34 & 4 \\ 32 & 52 \\ 36 \cdot 5 & 52 \\ 69 & 52\end{array}$


Table 1 (cont.)

Species

Staphylococcus cont.

$$
\text { saprophyticus (epidermidis) }
$$

saprophyticus

saprophyticus

saprophyticus

Streptococcus

bovis

cremoris

faecalis

faecalis

faecium

foetidus

liquefaciens

pneumoniae

pneumoniae

pyogenes

pyogenes

salivarius

sanguis

viridans

zymogenes

sp. Group G

sp. Group A

sp. Group A, L form

Streptomyces

albus

albus

albus

bobiliae

diastaticus

flaveolus

fradiae

fradiae

globisporus

griseolus

griseus

griseus

griseus

intermedius

kentuckensis

niveus

parvulus

parvus

streptomycini

venezuelae

violaceoruber

viridochromogenes

viridochromogenes

Streptosporangium

roseum

sp. (thermophilic)

Streptoverticillium cinnamomeus

Thermoactinopolyspora

sp.

sp.

Thermomonospora

sp.

sp.

\section{Strain}

Method

$\%$ GC

Ref.
$-$

7292

NCTC 7612

CCM 901

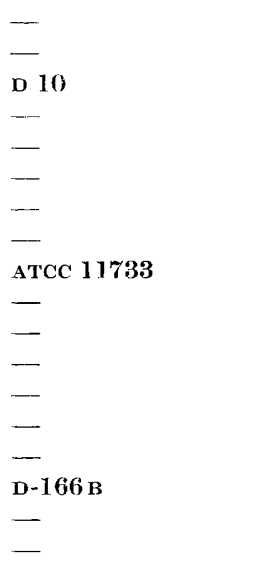

$\begin{array}{lll}\text { a } & 35 & 1 \\ \text { b } & 31 \cdot 5 & 52 \\ \text { b } & 31 & 52 \\ \text { bd } & 35 \cdot 5-39 & 4\end{array}$

$\begin{array}{lll}- & 38-40 & 38 \\ - & 38-4 \cdot 0 & 38 \\ \text { c } & 38 & 48 \\ \text { a } & 34 \cdot 5-38 \cdot 5 & 1 \\ - & 34-38 & 38 \\ \text { a } & 33 \cdot 5 & 1 \\ - & 34-38 & 38 \\ \text { a } & 38 \cdot 5 & 1 \\ \text { be } & 39-42 & 37,48 \\ \text { a } & 33 \cdot 5 & 1 \\ -- & 38-40 & 38 \\ \text { bc } & 39-42 & 37,48 \\ - & 38-40 & 38 \\ - & 38-40 & 38 \\ \text { a } & 33 \cdot 5 & 1 \\ \text { c } & 41 & 48 \\ \text { a } & 34 \cdot 5 & 44 \\ \text { a } & 33 \cdot 5 & 44\end{array}$

ATCC 618

1685

G

ATCC 3310

NRRL B-1270

ATCC 3319

ATCC 10745

3556

ATCC 3325

ATCC 10173

104

AтCC 3329

ATCC 12691

345

ATCC 12434

ATCC 12429

13

199

93

$27 \mathrm{~b}$

11

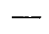

6

10

5 c

b

be

c

c

c

c

b

a

c

c

a

b

c

c

c

c

c

a

b

b

ab

be

$71 \cdot 5$

$71-74$

$70 \cdot 5$

$70 \cdot 5$

$70 \cdot 5$

$72.5-73.5$

$\begin{array}{ll}72 & 28\end{array}$

$73.5 \quad 1$

$70 \cdot 524$

$71 \cdot 524$

$73 \quad 1$

$69 \cdot 5 \quad 28$

$69.5 \quad 24$

69.524

$67 \quad 28$

$71.5 \quad 24$

$70 \cdot 524$

731

$69 \quad 28$

7128

$73-74 \quad 1,28$

$70-74 \quad 37,48$

$68.5 \quad 28$

49-53.5 13

$67 \quad 28$

b $\quad 6 r$

$77 \cdot 5 \quad 13$

$74 \quad 13$

b $\quad 74 \quad 13$

bc $\quad 44 \quad 13$ 


Species
Thiobacillus
novellus
thioparus
Treponema pallidum
Veillonella paroula
Vibrio
alcaligenes
anguillarum
(Campylobacter) bubulus
(Campylobacter) bubulus
(Campylobacter) bubulus
(Campylobacter) bubulus
cholinicus
comma (cholerae)
comma (cholerae)
comma (cholerae) and El Tor
costicolus
cuneatus
cuneatus
cyclocistes
(Campylobacter) fetus
(Campylobacter) fetus
(Campylobacter) fetus
ichthyodermis
marinus
marinus
metschnikovii
metschnikovii
neocistes
neocistes
percolans
percolans
percolans (Comamonas
terrigena)
succinogenes
tyrogenes
sp. (comma-like)
sp. (comma-like)
sp. (comma-like)
sp. (comma-like)
sp.
sp.
sp.
Xanthomonas
begoniae
campestris
carotae
carotae
hederae
hederae
juglandis
pelargonii
pelargonii
phaseoli
phaseoli
phaseoli
pisi
tamarindi
vesicatoria

Table 1 (cont.)

\begin{tabular}{|c|c|c|c|}
\hline Strain & Method & $\% \mathbf{G C}$ & Ref. \\
\hline CCM 1077 & bd & $58-59$ & 4 \\
\hline - & - & $68-70$ & 38 \\
\hline- & - & 34-38 & 38 \\
\hline - & $\mathbf{a}$ & 36.5 & 1 \\
\hline NCTC 9239 & $\mathbf{a}$ & 65 & 50 \\
\hline NCMB 6 & $\mathbf{a}$ & 44.5 & 50 \\
\hline IP 53103 & $\mathbf{a}$ & $29 \cdot 5$ & 50 \\
\hline IP 5421 & $\mathbf{a}$ & $29 \cdot 5$ & 50 \\
\hline IP 5422 & $\mathbf{a}$ & $30 \cdot 5$ & 50 \\
\hline IP 5420 & $\mathbf{a}$ & 31 & $\mathbf{5 0}$ \\
\hline 一 & 一 & $56-58$ & 38 \\
\hline- & $\mathbf{a}$ & $43 \cdot 5$ & $\mathbf{1}$ \\
\hline ATCC 9459 & bc & $47-49$ & $10,37,48$ \\
\hline (30 strains) & bc & $46-47$ & 12 \\
\hline NCMB 1001 & $\mathbf{a}$ & 50 & 50 \\
\hline ATCC 6972 & b & 61 & 9 \\
\hline NCIB 8194 & $\mathbf{a}$ & $62 \cdot 5$ & 50 \\
\hline NCIB 2581 & $\mathbf{a}$ & 64 & 50 \\
\hline IP 5396 & $\mathbf{a}$ & 33 & 50 \\
\hline IP 5443 & $\mathbf{a}$ & $34 \cdot 5$ & $\mathbf{5 0}$ \\
\hline IP 5395 & $\mathbf{a}$ & $35 \cdot 5$ & 50 \\
\hline NCMB 407 & $\mathbf{a}$ & 61 & 50 \\
\hline ATCC 15381 & c & 40 & 10 \\
\hline ATCC 15382 & c & 42 & 10 \\
\hline ATCC 7708 & bc & $43-43 \cdot 5$ & 9,10 \\
\hline IP A267 & $\mathbf{a}$ & 46 & 50 \\
\hline NCIB 2582 & $\mathbf{a}$ & 63.5 & 50 \\
\hline RH 1810 & bc & $63 \cdot 5-64.5$ & 8 \\
\hline ATCC 8461 & b & 65 & $\mathbf{9}$ \\
\hline NCTC 1937 & $\mathbf{a}$ & $64 \cdot 5$ & 50 \\
\hline
\end{tabular}

RH 260

ATCC 7085

IP 5638

NCTC 4711

NC'TC 4715

NCTC 8042

329

822

859

$61 \cdot 5-62 \cdot 5 \quad 8$

$49 \quad 50$

61.59

$45 \cdot 5 \quad 50$

4750

$47 \cdot 5 \quad 50$

$47 \cdot 5 \quad 50$

39110

$43 \quad 10$

$48 \quad 10$

B 3

C 129

C 104

D 230

XH 5

H 1

J 107

J.Koths

P 121

XP 163

XP 8

P 162

XP 171

T 20

v 136

$\begin{array}{ll}67 & 16 \\ 68 & 16 \\ 67 & 16 \\ 67 \cdot 5 & 16 \\ 62 & 9 \\ 67 & 16 \\ 66 & 16 \\ 62 \cdot 5 & 9 \\ 66 \cdot 5 & 16 \\ 63 & 9 \\ 63 & 9 \\ 66 & 16 \\ 64 & 9 \\ 67 \cdot 5 & 16 \\ 66 \cdot 5 & 16\end{array}$




\section{REFERENCES}

The references in this paper have been numbered for convenience and the numbers used in column 5 of the Table.

1. Belozersky, A. N. \& Spirin, A. S. (1960). The Nucleic Acids, Ed. by E. Chargaff and J. N. Davidson, vol. 3, p. 147. New York: Academic Press.

2. Bendich, A. (1957). Methods in Enzymology, Ed. by S. P. Colowick and N. O. Kaplan, New York: Academic Press.

3. Bergey's Manual of Determinative Bacteriology (1957), 7th ed. Ed. by R. S. Breed, E. G. D. Murray and N. R. Smith. London: Baillière, Tindall \& Cox.

4. Boháček, J., Kocur, M. \& Martinec, T. (1965). Deoxyribonucleic acid base composition and taxonomy of the genus Micrococcus. Publ. Fac. Sci., Univ., J. E. Purkinye Brno, K35, 318 (and personal communication).

5. Bouisset, L., Breuillaud, J. \& Michel, G. (1963). Étude de l'ADN chez les actinomycetales. Comparason entre les valeurs du rapport $A+T / G+C$ et les caractères bactérioliques des Corynebacterium. Ann. Inst. Pasteur 104, 756.

6. Cantoni, C., Hill, L. R. \& Silvestri, L. G. (1965). Deoxyribonucleic acid base composition of some members of the subgenera Betabacterium and Stretptobacterium Appl. Microbiol. 13, 631.

7. Catlin, B. W. \& Cunningham, L. S. (1961). Transforming activities and base contents of deoxyribonucleate preparations from various Neisseriae. J. gen. Microbiol. 26, 303.

8. Colwell, R. R., Citarelua, R. V. \& Ryman, I. (1965). Deoxyribonucleic acid base composition and Adansonian analysis of heterotrophic, aerobic pseudomonads. J. Bact. 90, 1148.

9. Colwell, R. R. \& Mandel, M. (1964). Adansonian analysis and deoxyribonucleic acid base composition of some gram-negative bacteria. $J$. Bact. 87, 1412 .

10. Colwell, R. R. \& Mandel, M. (1964). Base composition of deoxyribonucleic acid of marine and non-marine vibrios deduced from buoyant density measurements in cesium chloride. $J$. Bact. 88, 1816.

11. Colwell, R. R. \& Mandel, M. (1965). Adansonian analysis and deoxyribonucleic acid base composition of Serratia marcescens. J. Bact. 89, 454.

12. Colwell, R. R. \& Yuter, M. (1965). Adansonian analysis and deoxyribonucleic acid base composition studies on Vibrio cholerae and El Tor vibrios. Bact. Proc. G. 28.

13. Craveri, R., Hill, L. R., Manachini, P. L. \& Silvestri, L. G. (1965). Deoxyribonucleic acid base compositions among thermophilic actinomycetes: the occurrence of two strains with low GC content. J. gen. Microbiol. 41, 335 .

14. DE LEy, J. (1964). Effect of mutation on DNA-composition of some bacteria. Antonie van Leeurwenhoek 30, 281.

15. De Ley, J. (1964). Pseudomonas and related genera. A. Rev. Microbiol. $18,17$.

16. De Ley, J. \& van Muylem, J. (1963). Some applications of deoxyribonucleic acid base composition in bacterial taxonomy. Antonie van Leeuwenhoek 29, 344.

17. De Ley, J. \& Rassel, A. (1965). DNA base composition, flagellation and taxonomy of the genus Rhizobium. J. gen. Microbiol. 41, 85 .

18. De Ley, J. \& Schell, J. (1963). Deoxyribonucleic acid base composition of acetic acid bacteria. J. gen. Microbiol. 33, 243.

19. Dowell, V. R., Jr., Loper, J. C. \& Hill, E. O. (1964). Constancy of deoxyribonucleic acid base composition in the transition of Sphaerophorus necrophorus from bacilli to large bodies. $J$. Bact. 88, 1805 .

20. Dubnau, D., Smith, X., Morell, P. \& Marmur, J. (1965). Gene conservation in bacillus species, I. Conserved genetic and nucleic acid base sequence homologies. Proc. natn. Acad. Sci., U.S.A. 54, 491.

21. Enreinova, T. N., Bunina, N. V. \& Kusnetzova, N. Y. (1959). Biokhimiya 24, 912 (cited in refs. 37, 48).

22. Fedorova, L. S. (1964). Nucleotide composition of deoxyribonucleic and ribonucleic acids of the agent causing toxic bacteriosis of water lemons. Mikrobiologiya 33, 856. 
23. FredericQ, E., Oth, A. \& Fontaine, F. (1961). The ultraviolet spectrum of deoxyribonucleic acids and their constituents. J. molec. Biol. $3,11$.

24. Frontali, C., Hill, L. R. \& Silvestri, L. G. (1965). The base composition of deoxyribonucleic acids of Streptomyces. J. gen. Microbiol. 38, 243.

25. Gause, G. C., Loshkareva, N. P., Zbarsky, I. B. \& Gause, G. F. (1964). Deoxyribonucleic acid base composition in certain bacteria and their mutants with impaired respiration. Nature, Lond. 203, 598.

26. Jones, A. S., Titrensor, J. R. \& Walker, R. T. (1965). The chemical composition of the nucleic acids and other macromolecular constituents of Mycoplasma mycoides var. capri. J. gen. Microbiol. 40, 405.

27. Jones, A. S. \& Walker, R. T. (1963). Isolation and analysis of the deoxyribonucleic acid of Mycoplasma mycoides var. capri. Nature, Lond. 198, 588.

28. Jones, L. A. \& Bradley, S. G. (1964). Phenetic classification of actinomycetes. Devel. indust. Microbiol. 5, 267.

29. Joshi, J. G., Guild, W. R. \& Handler, P. (1963). The presence of two species of DNA in some halobacteria. $J$. molec. Biol. $6,34$.

30. Lynn, R. J. \& Smith, P. F. (1957). Nucleic acid content of pleuropneumonia-like organisms from human sources. J. Bact. 74, 811.

31. MacDonald, R. E. \& MacDonald, S. W. (1962). The physiology and natural relationships of the motile, spore-forming Sarcinae. Canad. J. Microbiol. 8, 795.

32. Mandel, M., Bergendahl, J. C. \& Prennig, N. (1965). Deoxyribonucleic acid base composition of isolates of the genus Chlorobium. J. Bact. 89, 917.

33. Mandel, M. \& Leadbetter, E. R. (1965). Deoxyribonucleic acid base composition of Myxobacteria. J. Bact. $90,1795$.

34. Mandel, M. \& Rowley, D. B. (1963). Configuration and base composition of deoxyribonucleic acid from spores of Bacillus subtilis var. niger. J. Bact. 85, 1445.

35. Mandel, M. \& Rownd, R. (1963). Taxonomic Biochemistry and Serology. Ed. by C. A. Leone, p. 585. New York: Ronald Press Co.

36. Mandel, M., Weeks, O. B. \& Colwell, R. R. (1965). Deoxyribonucleic acid base composition of Pseudomonas piscicida. J. Bact. 90, 1492.

37. Marmur, J. \& Doty, P. (1962). Determination of the base composition of deoxyribonucleic acid from its thermal denaturation temperature. J. molec. Biol. 5, 109.

38. Marmur, J., Falkow, S. \& Mandel, M. (1963). New approaches to bacterial taxonomy. Annu. Rev. Microbiol. 17, 329.

39. McCarthy, B. J. \& Bolton, E. T. (1963). An approach to the measurement of genetic relatedness among organisms. Proc. natn. Acad. Sci., U.S.A. $50,156$.

40. McDonald, W. C., Felkner, I. C., Turetsky, A. \& Matney, T. S. (1963). Similarity in base compositions of deoxyribonucleates from several strains of Bacillus cereus and Bacillus anthracis. J. Bact. 85, 1071.

41. Morowitz, H. J., Tourtellotte, M. E., Guild, W. R., Castro, E., Woese, C. \& Cleverdon, R. C. (1962). The chemical composition and submicroscopic morphology of Mycoplasma gallisepticum, Avian PPLO 5969. J. molec. Biol. 4, 93.

42. Moseley, B. E. B. \& Schein, A. H. (1964). Radiation resistance and deoxyribonucleic acid base composition of Micrococcus radiodurans. Nature, Lond. 203, 1298.

43. Neimark, H. C. \& PÈne, J. J. (1965). Characterization of pleuropneumonia-like organisms by deoxyribonucleic acid composition. Proc. Soc. exp. Biol. Med. 118, 517.

44. Panos, C. (1965). Cellular physiology during logarithmic growth of a streptococcal L-form. J. gen. Microbiol. 39, 131.

45. Poindexter, J. S. (1964). Biological properties and classification of the Caulobacter group. Bact. Rev. 28, 231.

46. Rogul, M., McGee, Z. A., Wittler, R. G. \& Falkow, S. (1965). Nucleic acid homologies of selected bacteria, $L$ forms and Mycoplasma species. J. Bact. 90, 1200.

47. Saunders, G. F., Campbell, L. L. \& Postgate, J. R. (1964). Base composition of deoxyribonucleic acid of sulfate-reducing bacteria deduced from buoyant density measurements in cesium chloride. J. Bact. 87, 1073. 
48. Schildkraut, C. L., Marmur, J. \& Doty, P. (1962). Determination of the base composition of deoxyribonucleic acid from its buoyant density in CsCl. $J$. molec. Biol. 4, 430.

49. Sebald, M., Gasser, F. \& Werner, H. (1965). Teneur GC \% et classification. Application au groupe des bifidobactéries et à quelques genres voisins. Ann. Inst. Pasteur $109,251$.

50. Sebald, M. \& Véron, M. (1963). Teneur en bases de l'ADN et classification des vibrions. Ann. Inst. Pasteur 105, 897.

51. Sigal, N., Senez, J. C., Le Gali, J. \& Sebald, M. (1963). Base composition of the deoxyribonucleic acid of sulphate-reducing bacteria. J. Bact. 85, 1315.

52. Silvestri, L. G. \& Hill, L. R. (1965). Agreement between deoxyribonucleic acid base composition and taxometric classification of gram-positive cocci.. $J$. Bact. 90, 136.

53. Sмгтн, J. D. \& Stoker, M. P. G. (1951). The nucleic acids of Rickettsia burneti. Br. J. exp. Path. $32,433$.

54. SueokA, N. (1961). Variation and heterogeneity of base composition of deoxyribonucleic acids: a compilation of old and new data. J. molec. Biol. 3, 31.

55. Tonomura, B., Malkin, R. \& Rabinowitz, J. C. (1965). Deoxyribonucleic acid base composition of clostridial species. J. Bact. 89, 1438.

56. WANG, S. Y. \& HAShageN, J. M. (1964). The determination of the base composition of deoxyribonucleic acids by bromination. $J$. molec. Biol. $8,333$.

57. WeEd, L. L. (1963). Effects of copper on Bacillus subtilis. J. Bact. 85, 1003.

58. Wyatt, G. R. \& Cohen, S. S. (1952). Nucleic acids of Rickettsiae. Nature, Lond. 170, 846. 